\title{
Deep molecular response predicts survival in $\mathrm{CML}$
}

New research has shown that a majority of patients with chronic myeloid leukaemia (CML) who are treated with the tyrosine kinase inhibitor imatinib can achieve a deep molecular response $(\geq 4.5-\log$ reduction in $B C R-A B L$ fusion gene transcript levels; $\mathrm{MR}^{4.5}$ ) and that $\mathrm{MR}^{4.5}$ is predictive of survival.

Major molecular response (MMR; $\geq 3-\log$ reduction in $B C R-A B L$ transcript levels) is currently used to assess prognosis. However, mounting evidence has shown that deeper molecular responses (that is, $\mathrm{MR}^{4.5}$ or higher) might correlate better with long-term outcomes. The CML-Study IV trial is the first to evaluate correlations between $\mathrm{MR}^{4.5}$ and long-term outcomes; molecular monitoring was performed for all patients in the study from the beginning.

More than 1,500 patients were enrolled and were assigned to several different imatinib-based regimens in terms of dosing or combination with other therapies (namely, IFN- $\alpha$, cytarabine or alone). The median observation time was 67.5 months.

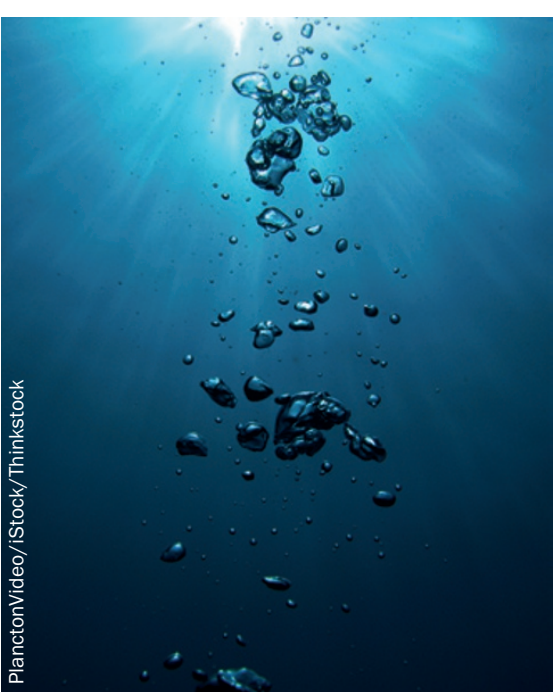

patients achieved $\mathrm{MR}^{4.5}$. Furthermore, this level of response was more common in patients who were treated with the highest doses of imatinib (800 mg per day, tolerability-optimized). "It is also interesting that $\mathrm{MR}^{4.5}$ is achieved faster in these patients than those receiving $400 \mathrm{mg}$ imatinib," comments Hehlmann. Additionally, $\mathrm{MR}^{4.5}$ at 4 years independently predicted survival and, in turn, high-dose imatinib and early MMR predicted $\mathrm{MR}^{4.5}$.

These results suggest that $\mathrm{MR}^{4.5}$ can be used to guide treatment cessation in patients with CML, with treatments selected that can achieve an early deep molecular response.

\section{Mina Razzak}

"Deep molecular response is a prestage of cure of CML," explains lead investigator Rüdiger Hehlmann, from the University of Heidelberg, Germany. "We found that a deep response level can be achieved in the majority of imatinib-treated patients with CML.” Indeed, after 9 years, $70 \%$ of
Original article Hehlmann, R. et al. Deep molecular response is reached by the majority of patients treated with imatinib, predicts survival, and is achieved more quickly by optimized high-dose imatinib: results from the randomized CML-Study IV. J. Clin. Oncol. doi:10.1200/ JCO.2013.49.9020 